\title{
Chimney and periscope grafts observed over 2 years after their use to revascularize 169 renovisceral branches in 77 patients with complex aortic aneurysms
}

\author{
Lachat, Mario ; Veith, Frank J ; Pfammatter, Thomas ; Glenck, Michael ; Bettex, Dominique ; Mayer,
}

Dieter ; Rancic, Zoran ; Gloekler, Steffen ; Pecoraro, Felice

\begin{abstract}
PURPOSE: To evaluate the performance of periscope and/or chimney grafts (CPGs) in the endovascular treatment of pararenal or thoracoabdominal aneurysms using off-the-shelf devices. METHODS: Between February 2002 and August 2012, 77 consecutive patients (62 men; mean age $73 \pm 9$ years) suffering from pararenal aortic $(n=55)$, thoracoabdominal $(n=16)$, or arch to visceral artery aneurysms $(n=6)$ were treated with aortic stent-graft implantation requiring chimney and/or periscope grafts to maintain side branch perfusion. CPGs were planned in advance and were not used as bailout. A standardized follow-up protocol including computed tomographic angiography, laboratory testing, and clinical examination was performed at 6 weeks; 3, 6, and 12 months; and annually thereafter. RESULTS: Technical success was achieved in 76 (99\%) patients; 1 branch stent-graft became dislocated from a renal artery, which could not be re-accessed. Overall, 169 target vessels (121 renal arteries, 30 superior mesenteric arteries, 17 celiac trunks, and 1 inferior mesenteric artery) were addressed with the chimney graft configuration in 111 and the periscope graft configuration in 58. In total, 228 devices were used for the CPGs: 213 Viabahn stent-grafts and 15 bare metal stents. Over a mean 25 16 months (range 1-121), 9 patients died of unrelated causes. Nearly all (95\%) of the patients demonstrated a decreased or stable aneurysm size on imaging; there was a mean $13 \%$ shrinkage in aneurysm diameter. Twenty patients had primary type I/III endoleaks at discharge; in follow-up, only 3 of these were still present (no secondary or recurrent endoleaks were noted). Additional endovascular maneuvers were required for CPG-related complications in 13 patients from intervention throughout follow-up. Overall, 4 CPGs occluded (98\% target vessel patency); no stent-graft migration was observed. Renal function remained stable in all patients. CONCLUSION: In this series, the use of CPGs has proven to be a feasible, safe, and effective way to treat thoracoabdominal and pararenal aneurysms with maintenance of blood flow to the renovisceral arteries. Nearly all of the aneurysms showed no increase in diameter over a $>2$-year mean follow-up, which supports the midterm adequacy of the CPG technique as a method to effectively revascularize branch vessels with few endoleaks or branch occlusions.
\end{abstract}

DOI: https://doi.org/10.1583/13-4372.1

Posted at the Zurich Open Repository and Archive, University of Zurich

ZORA URL: https://doi.org/10.5167/uzh-85121

Journal Article

Published Version

Originally published at: 
Lachat, Mario; Veith, Frank J; Pfammatter, Thomas; Glenck, Michael; Bettex, Dominique; Mayer, Dieter; Rancic, Zoran; Gloekler, Steffen; Pecoraro, Felice (2013). Chimney and periscope grafts observed over 2 years after their use to revascularize 169 renovisceral branches in 77 patients with complex aortic aneurysms. Journal of Endovascular Therapy, 20(5):597-605.

DOI: https://doi.org/10.1583/13-4372.1 


\title{
Chimney and Periscope Grafts Observed Over 2 Years After Their Use to Revascularize 169 Renovisceral Branches in 77 Patients With Complex Aortic Aneurysms
}

\author{
Mario Lachat, MD'; Frank J. Veith, MD $^{2}$; Thomas Pfammatter, $\mathrm{MD}^{3}$; Michael Glenck, $\mathrm{MD}^{3}$; \\ Dominique Bettex, MD ${ }^{4}$; Dieter Mayer, MD ${ }^{1}$; Zoran Rancic, MD, PhD ${ }^{1}$; Steffen Gloekler, MD ${ }^{5}$; \\ and Felice Pecoraro, MD ${ }^{6}$ \\ ${ }^{1}$ Clinic for Cardiovascular Surgery, ${ }^{3}$ Interventional Radiology, and ${ }^{4}$ Division of Cardiovascular \\ Anesthesia, Institute of Anesthesiology, University Hospital, Zurich, Switzerland. ${ }^{2}$ New York \\ University Medical Center, New York, New York, and The Cleveland Clinic, Cleveland, Ohio, USA. \\ ${ }^{5}$ Department of Cardiology, University Hospital, Bern, Switzerland. ${ }^{6}$ Vascular Surgery Unit, \\ University of Palermo, AOUP "P. Giaccone" Palermo, Italy.
}

Purpose: To evaluate the performance of periscope and/or chimney grafts (CPGs) in the endovascular treatment of pararenal or thoracoabdominal aneurysms using off-the-shelf devices.

Methods: Between February 2002 and August 2012, 77 consecutive patients $(62$ men; mean age $73 \pm 9$ years) suffering from pararenal aortic $(n=55)$, thoracoabdominal $(n=16)$, or arch to visceral artery aneurysms $(n=6)$ were treated with aortic stent-graft implantation requiring chimney and/or periscope grafts to maintain side branch perfusion. CPGs were planned in advance and were not used as bailout. A standardized follow-up protocol including computed tomographic angiography, laboratory testing, and clinical examination was performed at 6 weeks; 3, 6, and 12 months; and annually thereafter.

Results: Technical success was achieved in 76 (99\%) patients; 1 branch stent-graft became dislocated from a renal artery, which could not be re-accessed. Overall, 169 target vessels (121 renal arteries, 30 superior mesenteric arteries, 17 celiac trunks, and 1 inferior mesenteric artery) were addressed with the chimney graft configuration in 111 and the periscope graft configuration in 58. In total, 228 devices were used for the CPGs: 213 Viabahn stent-grafts and 15 bare metal stents. Over a mean $25 \pm 16$ months (range 1-121), 9 patients died of unrelated causes. Nearly all (95\%) of the patients demonstrated a decreased or stable aneurysm size on imaging; there was a mean $13 \%$ shrinkage in aneurysm diameter. Twenty patients had primary type $\mathrm{I} / \mathrm{III}$ endoleaks at discharge; in follow-up, only 3 of these were still present (no secondary or recurrent endoleaks were noted). Additional endovascular maneuvers were required for CPG-related complications in 13 patients from intervention throughout follow-up. Overall, 4 CPGs occluded (98\% target vessel patency); no stent-graft migration was observed. Renal function remained stable in all patients.

Conclusion: In this series, the use of CPGs has proven to be a feasible, safe, and effective way to treat thoracoabdominal and pararenal aneurysms with maintenance of blood flow to the renovisceral arteries. Nearly all of the aneurysms showed no increase in diameter over a $>2$ year mean follow-up, which supports the midterm adequacy of the CPG technique as a method to effectively revascularize branch vessels with few endoleaks or branch occlusions.

J Endovasc Ther. 2013;20:597-605

Presented as an abstract at the 2012 Vascular Annual Meeting held in National Harbor, Maryland, USA, on June 7-9, 2012.

The authors declare no association with any individual, company, or organization having a vested interest in the subject matter/products mentioned in this article.

Corresponding author: Felice Pecoraro, MD, Vascular Surgery Unit, AOUP "P. Giaccone" Palermo, Via Liborio Giuffre, 90100 Palermo, Italy. E-mail: felicepecoraro@libero.it 
Key words: aortic aneurysm, pararenal aortic aneurysm, thoracoabdominal aortic aneurysm, aortic arch, endovascular aneurysm repair, renal artery, superior mesenteric artery, celiac trunk, target vessel, stent-graft, side branch, chimney graft, periscope graft, parallel graft, target vessel patency, endoleak, side branch occlusion

Renal artery stent placement to rescue an inadvertently covered renal artery orifice during aortic stent-graft deployment is a well-known bailout technique first reported by Greenberg et al. ${ }^{1}$ and Larzon and colleagues. ${ }^{2}$ Later, the term "chimney graft" was proposed when covered stents were deployed for this purpose. ${ }^{3}$ More recently, the "periscope graft" technique was described, ${ }^{4}$ which

See commentary page 606

is similar to the chimney technique but in reverse fashion to extend the distal stent-graft landing zone. Use of chimney and periscope grafts was also reported to treat ruptured thoracoabdominal aortic aneurysms (TAAA) successfully. 5,6

The majority of the published case series present the short-term results of small groups of patients treated with a single bare metal or covered stent placed as a chimney graft. ${ }^{7}$ The aim of the present article is to report our ongoing experience with 77 consecutive patients treated over a 10-year period with renovisceral chimney and/or periscope grafts (CPGs).

\section{METHODS}

\section{Study Cohort}

From June 2002 to August 2012, 77 consecutive high-risk patients (62 men; mean age $73 \pm 9$ years) with aortic aneurysms extending to or involving the visceral aorta were treated with aortic stent-graft implantation and CPGs. All patients were considered at high risk for open aortic surgery and/or a standard renovisceral debranching procedure. ${ }^{8}$ Fourteen were $\geq 80$ years old. Patient morbidity included coronary artery or valvular heart disease and/or a left ventricular ejection fraction $<25 \%$, chronic obstructive pulmonary disease, a hostile chest or abdomen (after prior abdominal or aortic surgery), and reduced renal function (Table 1). More than half $(48$, $62 \%)$ presented with multiple comorbidities. Mean glomerular filtration rate (GFR) at baseline was $54 \pm 10 \mathrm{~mL} / \mathrm{min} / 1.73 \mathrm{~m}^{2}$. Nine patients were treated for a ruptured aneurysm and 12 for a symptomatic aneurysm. Mean aneurysm diameter was $70 \pm 23 \mathrm{~mm}$ (range 44-185). Fifty-five (71.4\%) patients presented with a pararenal aortic aneurysm, $16(20.8 \%)$ patients with a TAAA, and $6(7.8 \%)$ patients with an arch to visceral aortic aneurysm.

A preoperative thoracoabdominal computed tomographic angiography (CTA) was performed in all patients to assess anatomical details, plan the operative strategy, and measure maximum aneurysm diameter perpendicular to the flow line. ${ }^{9}$ CPGs were planned in advance in all patients and were not used as a bailout after unintentional coverage of an aortic branch.

Patient imaging data, physiological parameters, and outcome data were collected prospectively in the clinical information system (Dendrite and KISIM 4.901; Dendrite Clinical Systems, Ltd., Henley-on-Thames,

\begin{tabular}{lc}
\hline \multicolumn{2}{c}{ TABLE 1 } \\
\multicolumn{2}{c}{$\begin{array}{c}\text { Comorbidities of 77 Patients Treated With } \\
\text { Chimney and/or Periscope Grafts }\end{array}$} \\
\hline $\begin{array}{cc}\text { Age, y } \\
\geq 80 \text { years old }\end{array}$ \\
Men & $73 \pm 9$ \\
Cardiac disease & 14 \\
COPD & 62 \\
Decreased renal function & $43(56 \%)$ \\
Cerebrovascular disease & $28(36 \%)$ \\
Hostile chest/abdomen & $12(16 \%)$ \\
PAD & $49(63 \%)$ \\
\hline
\end{tabular}

Continuous data are presented as the means \pm standard deviation; categorical data are given as the counts (percentage).

COPD: chronic obstructive pulmonary disease, PAD: peripheral artery disease. 
UK) of our institution and analyzed retrospectively in February 2013. Informed consent for the procedure itself and the anonymous data collection and analysis was obtained from all patients. Early data on some of these patients $\left(35^{10}\right.$ and $\left.26^{11}\right)$ have been published as part of dual institution reports.

\section{Technique}

All procedures were performed in an angiography suite equipped for surgical interventions or a hybrid operating room with the patients under local $(n=22)$ or general $(n=55)$ anesthesia. Based on the anatomy of the aortic aneurysm and the renovisceral target arteries, a tailored approach for each patient was predefined on the basis of the CTA. In case of multiple CPGs, both axillary and femoral accesses were required to address the target vessels (Fig. 1).

Five commercially available stent-grafts [Excluder and TAG (W.L. Gore \& Associates, Flagstaff, AZ, USA); Evita (Jotec, Hechingen, Germany); Talent (Medtronic Vascular, Santa Rosa, CA, USA); and Zenith (Cook Inc., Bloomington, IN, USA)] were employed and tailored according to the CTA data. Diameter sizing of the aortic stent-graft was based on the mean aortic diameter and the mean diameter of the CPGs. For 2 CPGs, the mean diameter of the CPGs was added to the mean diameter of the aortic neck at the graft fixation site. If $3 \mathrm{CPGs}$ were used, $1.5 \times$ the mean diameter of each CPG was added to the mean aortic diameter.

Remote access for aortic stent-graft introduction/deployment was mostly via the femoral artery and, in some rare cases, the aorta or iliac artery. Renovisceral branch cannulation was generally achieved using a $45-\mathrm{cm}$ Arrow sheath (Arrow International Inc., Reading, PA, USA), a 5-F Chuang visceral reverse curve catheter (Cook Inc.), and a Rosen wire (Cook Inc.). The same procedure was repeated for each renovisceral branch. After cannulation, the chimney or periscope graft was positioned in the target artery and deployed with the distal end $\sim 2 \mathrm{~cm}$ into the target vessel. These parallel grafts were mostly the Viabahn (W.L. Gore \& Associates); sometimes bare metal stents were used [Wallstent (Bos-

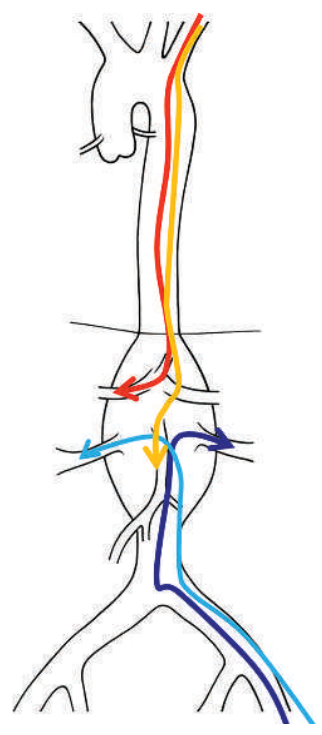

Figure $1 \diamond$ Multiple chimney/periscope graft diagram: access to the celiac trunk and superior mesenteric artery from the left subclavian artery in the chimney configuration; access to the renal arteries from the left femoral artery in a periscope configuration.

ton Scientific Corporation, Natick, MA, USA) and Palmaz Blue or Corinthian SES (Cordis Endovascular/Johnson \& Johnson, Miami, FL, USA)]. To achieve safe anchoring, angioplasty balloons $(2 \mathrm{~cm}$ in length) were used to fully expand the CPGs at their anchoring/landing zone. The deflated balloons remained in the target arteries in order to prevent the CPGs from crossing over each other at the level of the aortic stent-graft landing zone. During deployment of the CPGs, the balloons were inflated in the target vessels and pullback traction was applied on the balloon catheters to keep the branch endografts straight while deploying the aortic stent-graft. A kissing balloon technique completed the procedure by achieving full and simultaneous expansion of all the different stent-grafts used, i.e., aortic, periscope, and chimney. In a ruptured Crawford II TAAA, the renal periscope endografts were deployed using a sandwich technique. ${ }^{12}$

Once all devices were implanted, completion angiography, as well as selective pressure measurements distal to the aortic and any branch devices, was performed to exclude endoleaks and/or significant pressure 


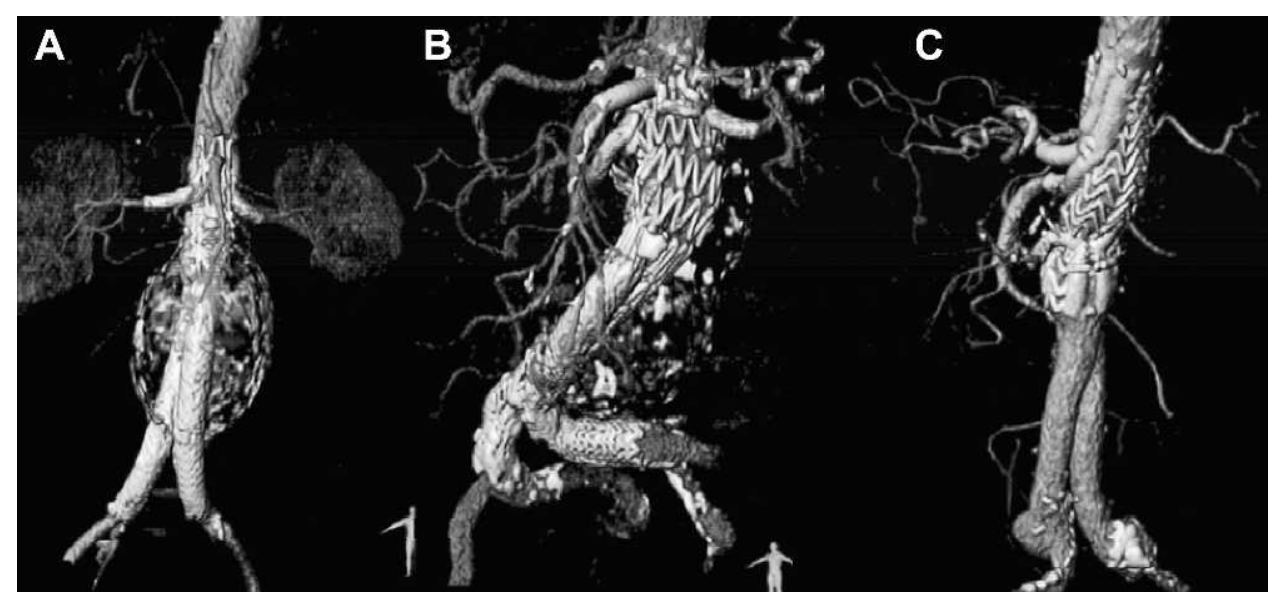

Figure $2 \diamond$ Chimney and/or periscope graft CTA 3D reconstruction. (A) Double renal artery chimney grafts. (B) Triple chimney grafts in the renal arteries and superior mesenteric artery. (C) Complete endodebranching: double chimney grafts in the SMA and celiac trunk and double periscope grafts in the renal arteries. These CTAs were obtained at 12, 24, and 36 months, respectively, after the procedures.

gradients. Any significant high-flow type I or III endoleak detected during contrast injection was corrected immediately. In case of a significant pressure drop in a target vessel, additional kissing balloon inflation and/or bare metal stenting were performed.

Immediately after the procedure, duplex sonography was performed to confirm physiological perfusion of the abdominal organs and the absence of significant iliofemoral flow obstruction. A CTA was performed before hospital discharge in all patients. Any significant high-flow type I, II, or III endoleak filling whole or part of the aneurysm sac in the arterial phase of the CTA was corrected. Lowflow type I endoleaks emerging only during late-phase CTA and low-flow type II endoleaks were managed conservatively. ${ }^{10}$

\section{Follow-up Protocol}

Perioperative antiplatelet therapy $(100 \mathrm{mg} / \mathrm{d}$ aspirin) combined with full heparinization was administered. Before discharge, patients were switched to aspirin and an oral anticoagulant for at least 3 months or to a dual antiplatelet regimen (aspirin and clopidogrel). A standardized follow-up protocol including CTA, laboratory testing, and clinical examination was performed at 6 weeks; 3, 6, and 12 months; and annually thereafter. Postoperative CTA was employed to assess changes in aneurysm diameter. ${ }^{9}$ If there was a contraindication to contrast agent injection, a non-contrast CT and a duplex ultrasound of the aorta and target vessels were performed.

\section{RESULTS}

The aortic aneurysms were treated with 46 bifurcated, 30 straight, and 1 aortouni-iliac stent-graft configurations using a mean of 3 stent-grafts (median 2, range 1-5) in each patient. The mean diameter of the stent-grafts was $32.0 \pm 4.5 \mathrm{~mm}$ (range 23-46), while the mean aortic diameter neck was $28.2 \pm 4.4 \mathrm{~mm}$ (range 19-38).

Immediate technical success (procedure completed as intended) was achieved in 76 (99\%) patients (Fig. 2). In one obese patient (body mass index $41 \mathrm{~kg} / \mathrm{m}^{2}$ ) presenting with a ruptured pararenal aneurysm, a branch stentgraft became dislocated from the right renal artery. This artery could not be re-accessed, and the right kidney was sacrificed.

Overall, 169 target vessels (121 renal arteries, 30 superior mesenteric arteries, 17 celiac trunks, and 1 inferior mesenteric artery) were addressed using the chimney graft configuration in 111 and the periscope graft configuration in 58. In total, 228 devices were employed to construct the CPGs: 213 Viabahn stent- 
TABLE 2

Chimney/Periscope Graft (CPG) Configurations and Devices Used With Respect to Location

\begin{tabular}{|c|c|c|c|c|c|c|c|}
\hline & \multirow[b]{2}{*}{$\mathrm{N}$} & \multicolumn{2}{|c|}{ CPG Configuration } & \multicolumn{2}{|c|}{ CPG Stent Types } & \multicolumn{2}{|c|}{ CPG Dimensions } \\
\hline & & Chimney & Periscope & Covered & Bare & Diameter, mm & Length, $\mathrm{cm}$ \\
\hline RRA & 60 & 34 & 26 & 80 & 3 & $6.47 \pm 0.79$ & $7.02 \pm 3.17$ \\
\hline LRA & 50 & 31 & 19 & 80 & 3 & $6.66 \pm 0.89$ & $6.72 \pm 2.71$ \\
\hline SMA & 30 & 23 & 7 & 80 & 5 & $8.18 \pm 1.10$ & $5.86 \pm 2.42$ \\
\hline CT & 16 & 11 & 5 & 21 & 4 & $8.24 \pm 1.20$ & $7.43 \pm 4.67$ \\
\hline IMA & 1 & 0 & 1 & 1 & 0 & 7 & 5 \\
\hline
\end{tabular}

Continuous data are presented as the means \pm standard deviation; categorical data are given as the counts. RRA: right renal artery, LRA: left renal artery, SMA: superior mesenteric artery, CT: celiac trunk, IMA: inferior mesenteric artery.

grafts and 15 bare metal stents (mean 1.35/ vessel; 2.19/patient). Table 2 describes the CPG configuration and devices used with respect to the location. The mean procedure time, including surgical access and skin closure, was $248 \pm 104$ minutes (range 90390 ) with a mean radiation dose area product of $45494 \pm 29022 \mathrm{mGy}^{*} \mathrm{~cm}^{2}$ (range 1447397899).

The mean lengths of stay were $2.2 \pm 4.1$ days in intensive care and $14 \pm 20$ days in hospital. One patient with a ruptured pararenal aneurysm died from reperfusion injury and multiple organ failure on the $9^{\text {th }}$ postoperative day. Another patient with a ruptured pararenal aneurysm died on the $7^{\text {th }}$ postoperative day from multiorgan failure. Perioperative major complications included a renal hematoma in 3 patients and a psoas hematoma due to an iliac rupture. The renal hematomas were treated conservatively; the iliac rupture was excluded with the bifurcated stent-graft during the initial endovascular repair. Perioperative access site complications included 2 left axillary hematomas treated with surgical evacuation and a bilateral lymphatic fistula treated with vacuum-assisted closure therapy.

At postoperative CTA, 19 patients had primary type I [16 la and $3 \mathrm{lb}$ (Fig. 3)] endoleaks, 1 patient had a type III endoleak, and 14 patients had type II leaks (2 patients presented both types la and II). At a mean follow-up of $25.5 \pm 16.0$ months (range 1-121), 3 of the 20 primary type $\mathrm{I} / \mathrm{II}$ endoleaks were still present, but no secondary or recurrent endoleaks were noted. Additional endovascu- lar maneuvers (Fig. 4) were required for CPGrelated complications in 13 patients from intervention throughout follow-up; in 1 case this procedure was planned intraoperatively.

Mean aortic aneurysm diameter at followup decreased by $13.3 \% \pm 20.0 \%$ to $61.1 \mathrm{~mm}$ (range 30-160). In $4(5.2 \%)$ patients, an increase in the aneurysm diameter was observed; one of these patients had a type la endoleak, and an endovascular perigraft embolization is planned.

During follow-up, no statistically significant difference was observed in renal function (mean GFR $56.2 \pm 11.0 \mathrm{~mL} / \mathrm{min} / 1.72 \mathrm{~m}^{2}$ ). Over the course of the study period, 9 patients died of unrelated causes (actuarial patient survival is presented in Figure 5A). From intervention onward, 4 CPGs occluded $(98 \%$ target vessel patency), all within 30 days. The Kaplan-Meier freedom from occlusion estimate is presented in Figure 5B.

\section{DISCUSSION}

Open graft repair of pararenal, thoracoabdominal, and aortic aneurysms generally has a substantial operative mortality and morbidity. ${ }^{1,13}$ Endovascular repair with fenestrated and/or branched stent-grafts is an attractive alternative to open repair, but has significant limitations. Although some have reported successful use of surgeon-made fenestrated and branched endografts, ${ }^{14}$ the commercial devices generally have to be customized, which excludes urgent or emergent patients. In addition, some patients are unsuitable for 

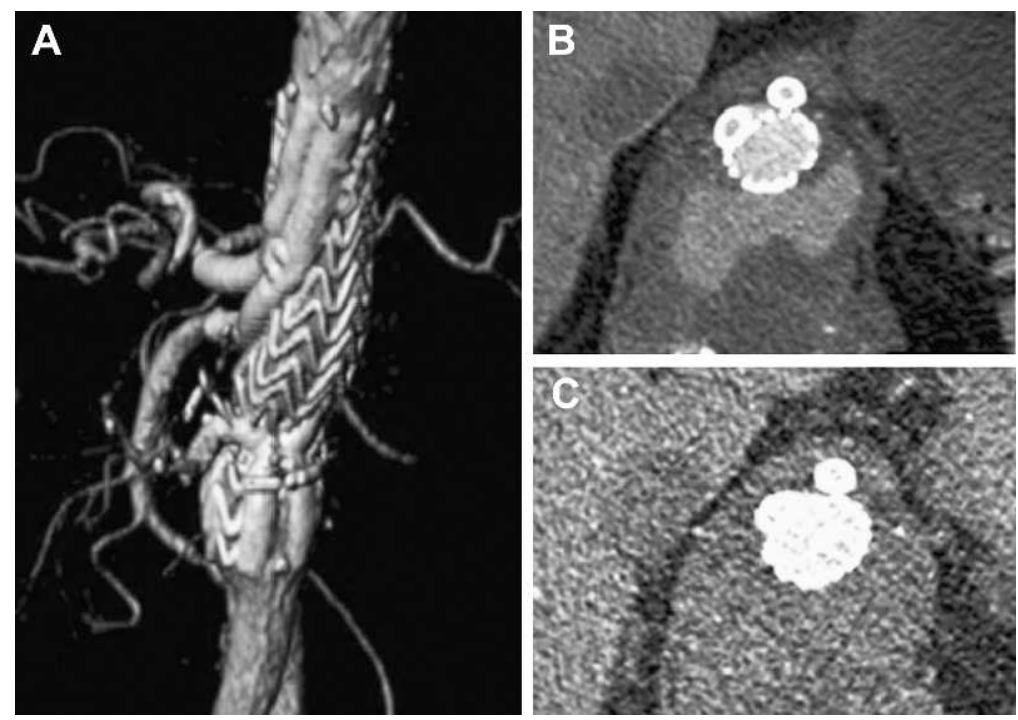

Figure $3-(\mathbf{A}, \mathbf{B})$ Postoperative CTA showing "low-flow" type la endoleak associated with renal artery chimney grafts. (C) At 1 month there has been spontaneous sealing of the endoleak.

any of these devices. ${ }^{15,16}$ On the other hand, CPGs are an alternative way of treating these complex aneurysms and allow the use of offthe-shelf devices to treat emergency cases, patients unfit for thoracoabdominal open surgery, or patients who are not suitable for branched or fenestrated stent-grafts due to anatomical restrictions. ${ }^{10,16-20}$

CPGs have some major advantages over the branched graft techniques. For each aortic branch, the best branch design, chimney or periscope, can be chosen specifically based on the target vessel, the remote access vessel, and the aortic anatomy so that the procedure can be performed without delay. In addition, navigation and/or manipulations are greatly simplified as the wires, catheters, and parallel stent-grafts do not have to be directed through the aortic stent-graft. An additional advantage of the CPGs is that they minimize
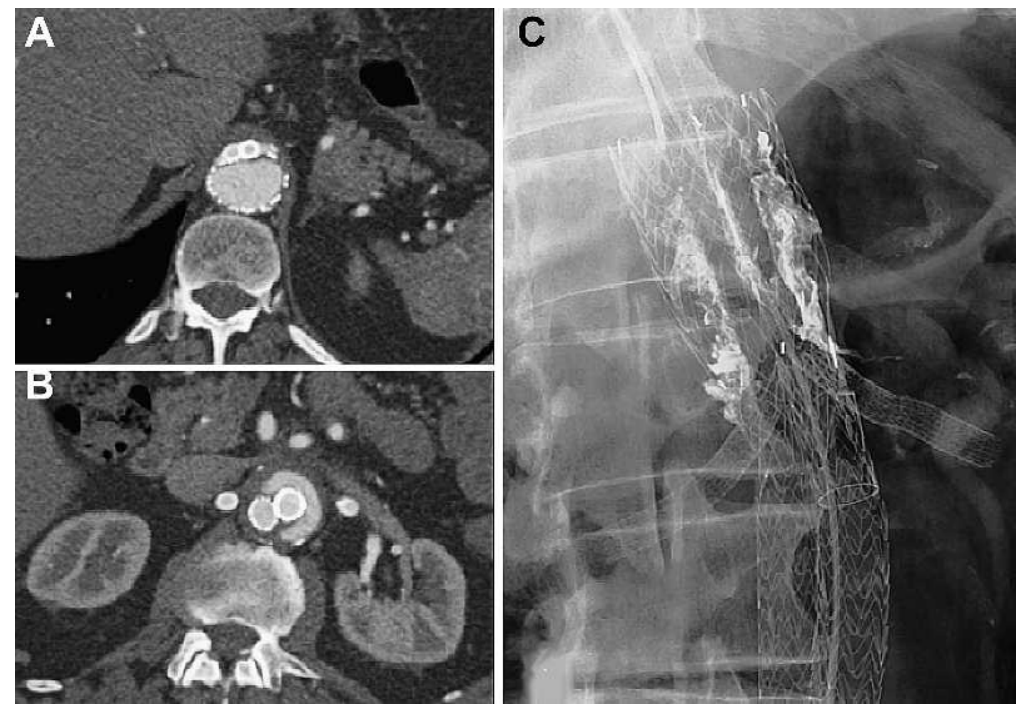

Figure $4 \diamond(\mathbf{A}, \mathbf{B})$ Postoperative CTA showing "high-flow" type la endoleak associated with renal artery chimney grafts. (C) Intraoperative angiogram showing perileak embolization with Onyx. 
A

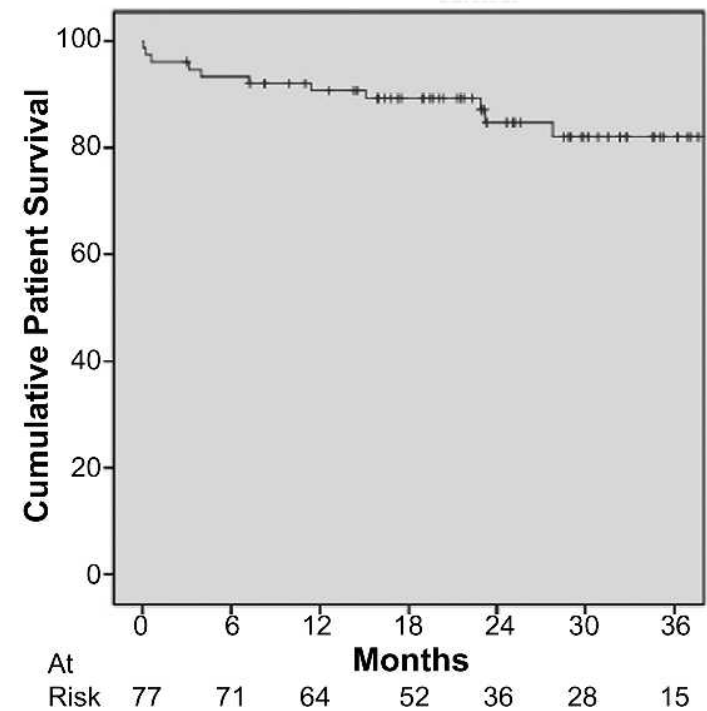

B

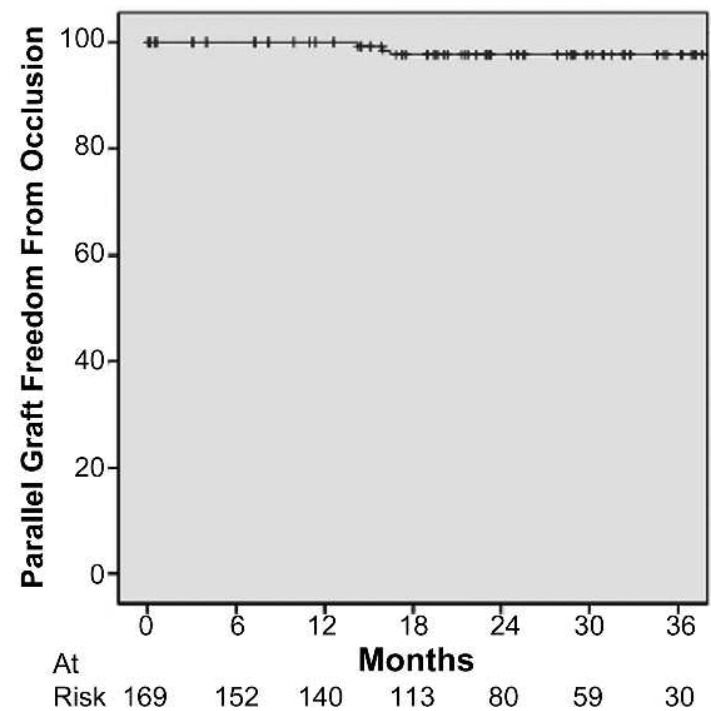

Figure 5 (A) Cumulative survival of patients treated with chimney and/or periscopes grafts and (B) freedom from occlusion for the parallel grafts. Standard error did not exceed $10 \%$ at 36 months.

secondary stent-graft dislocation out of the aortic branch, as the CPGs are fixed inbetween the aortic wall and the aortic stentgraft.

Endoleaks have been reported in association with CPGs. ${ }^{20}$ We differentiate two types of primary endoleaks: "high-flow," which appear immediately after contrast injection, vs. "low-flow," which appear after some delay. We treat all primary and secondary high-flow types la and lb leaks but tend to observe low-flow ones. ${ }^{10}$ Even in patients with ruptured aneurysms and low-flow leaks, we have observed that hemorrhage is effectively controlled. Moreover, low-flow leaks encountered in patients with severely disturbed coagulation usually disappear once the coagulation system normalizes. Using covered stent-grafts (such as the Viabahn) for the periscope or chimney grafts usually prevents a type $\mathrm{I} / \mathrm{III}$ endoleak.

To avoid high-flow type I leaks along the CPGs gutters, we have developed and used a method to adequately size the aortic stentgraft. Adding half of the mean diameter of all the CPGs used to the mean aortic diameter leads to adequate main stent-graft redundancy to fully cover all the CPGs. The overlap length between the CPGs and the main aortic graft is another important issue. To achieve adequate sealing between the CPGs, the main aortic graft, and the aortic wall, an overlap of $\sim 4 \mathrm{~cm}$ is required to minimize type I endoleaks and gutters, although this may not always be anatomically feasible.

However, there are some general limitations to this technique. For the construction of CPGs, access through the subclavian artery and the aortic arch has to be safe. Any floating plaques or arch disease in the region of the supra-aortic trunks carries a high stroke risk and represent a contraindication to placement of CPGs from above. There are, however, methods to approach visceral arteries from the femoral arteries to get a chimney configuration. ${ }^{21}$ In addition, some degree of branch disease can be accepted with this technique, but the target artery should not have major disease and should have a minimal diameter of $>4 \mathrm{~mm}$ and a safe landing zone of $>1 \mathrm{~cm}$. In addition, the CPG technique requires highend imaging tools. Especially with multiple CPGs, clear visualization of catheters, wires, sheaths, and grafts is essential, and standard portable C-arm fluoroscopy may not be adequate. Finally, although the present midterm results, along with other reports, ${ }^{11}$ are encouraging and suggest that CPGs are a 
durable way of revascularizing renovisceral aortic branches involved in aneurysmal disease, the overall experience with CPGs is limited, and long-term follow-up is needed to document the durability of these techniques.

\section{Conclusion}

In this series, the use of CPGs has proven to be a feasible, safe, and effective way to treat thoracoabdominal and pararenal aneurysms with maintenance of blood flow to the renovisceral arteries. Nearly all of the aneurysms showed no increase in diameter and a $13 \%$ average aneurysm diameter reduction over a mean $>2$-year follow-up, which supports the midterm adequacy of the CPG technique as a method to effectively revascularize branch vessels with few endoleaks or branch occlusions. Thus, provided certain technical requirements are fulfilled, these techniques appear to be justified when patients are at high risk for other methods of repair or have complex ruptured aneurysms involving vital aortic branch arteries.

\section{References}

1. Greenberg RK, Clair D, Srivastava S, et al. Should patients with challenging anatomy be offered endovascular aneurysm repair? J Vasc Surg. 2003;38:990-996.

2. Larzon T, Eliasson K, Gruber G. Top-fenestrating technique in stent-grafting of aortic diseases with mid-term follow-up. J Cardiovasc Surg (Torino). 2008;49:317-322.

3. Criado FJ. A percutaneous technique for preservation of arch branch patency during thoracic endovascular aortic repair (TEVAR): retrograde catheterization and stenting. J Endovasc Ther. 2007;14:54-58.

4. Rancic Z, Pfammatter $T$, Lachat $M$, et al. Periscope graft to extend distal landing zone in ruptured thoracoabdominal aneurysms with short distal necks. J Vasc Surg. 2010;51:12931296.

5. Lachat M, Frauenfelder T, Mayer D, et al. Complete endovascular renal and visceral artery revascularization and exclusion of a ruptured type IV thoracoabdominal aortic aneurysm. J Endovasc Ther. 2010;17:216-220.

6. Pecoraro F, Pfammatter T, Mayer D, et al. Multiple periscope and chimney grafts to treat ruptured thoracoabdominal and pararenal aor- tic aneurysms. J Endovasc Ther. 2011;18:642649.

7. Moulakakis KG, Mylonas SN, Avgerinos E, et al. The chimney graft technique for preserving visceral vessels during endovascular treatment of aortic pathologies. J Vasc Surg. 2012;55: 1497-1503.

8. Chaikof EL, Fillinger MF, Matsumura JS, et al. Identifying and grading factors that modify the outcome of endovascular aortic aneurysm repair. J Vasc Surg. 2002;35:1061-1066.

9. Chaikof EL, Blankensteijn JD, Harris PL, et al. Reporting standards for endovascular aortic aneurysm repair. J Vasc Surg. 2002;35:10481060.

10. Donas KP, Pecoraro F, Torsello G, et al. Use of covered chimney stents for pararenal aortic pathologies is safe and feasible with excellent patency and low incidence of endoleaks. J Vasc Surg. 2012;55:659-665.

11. Donas KP, Pecoraro F, Bisdas T, et al. CT angiography at 24 months demonstrates durability of EVAR with the use of chimney grafts for pararenal aortic pathologies. J Endovasc Ther. 2013;20:1-6.

12. Kolvenbach RR, Yoshida R, Pinter $L$, et al. Urgent endovascular treatment of thoracoabdominal aneurysms using a sandwich technique and chimney grafts-a technical description. Eur J Vasc Endovasc Surg. 2011;41:54-60.

13. Derrow AE, Seeger JM, Dame DA, et al. The outcome in the United States after thoracoabdominal aortic aneurysm repair, renal artery bypass, and mesenteric revascularization. J Vasc Surg. 2001;34:54-61.

14. Oderich GS, Ricotta JJ. Modified fenestrated stent grafts: device design, modifications, implantation, and current applications. Perspect Vasc Surg Endovasc Ther. 2009;21:157-167.

15. Greenberg RK. Aortic aneurysm, thoracoabdominal aneurysm, juxtarenal aneurysm, fenestrated endografts, branched endografts, and endovascular aneurysm repair. Ann NY Acad Sci. 2006;1085:187-196.

16. Verhoeven EL, Vourliotakis G, Bos WT, et al. Fenestrated stent grafting for short-necked and juxtarenal abdominal aortic aneurysm: an 8year single-centre experience. Eur $J$ Vasc Endovasc Surg. 2010;39:529-536.

17. Chuter TA, Gordon RL, Reilly LM, et al. An endovascular system for thoracoabdominal aortic aneurysm repair. J Endovasc Ther. 2001;8:25-33.

18. Lee JT, Greenberg JI, Dalman RL. Early experience with the snorkel technique for juxtarenal aneurysms. J Vasc Surg. 2012;55:935-946. 
19. Amiot S, Haulon S, Becquemin JP, et al. Fenestrated endovascular grafting: the French multicentre experience. Eur J Vasc Endovasc Surg. 2010;39:537-544.

20. Hiramoto JS. Commentary: multiple chimney grafts for total endovascular revascularization of the visceral arteries in the setting of ruptured
TAAA: inventive but let's wait for the smoke to clear on this one. J Endovasc Ther. 2010;17: 222-223.

21. Lachat M, Bisdas $T$, Rancic $Z$, et al. Chimney endografting for pararenal aortic pathologies using transfemoral access and the lift technique. J Endovasc Ther. 2013;20:492-497. 